\title{
A Taxonomy for Combining Activity Recognition and Process Discovery in Industrial Environments
}

\author{
Felix Mannhardt ${ }^{1}[0000-0003-1733-777 X]$, Riccardo Bovo $^{2}$, Manuel Fradinho Oliveira ${ }^{1}$, \\ and Simon Julier ${ }^{2}[0000-0003-4380-137 X]$ \\ 1 SINTEF Digital, Trondheim, Norway \\ firstname.lastname@sintef.no \\ 2 Department of Computer Science, UCL, London, United Kingdom \\ firstname.lastname@ucl.ac.uk
}

\begin{abstract}
Despite the increasing automation levels in an Industry 4.0 scenario, the tacit knowledge of highly skilled manufacturing workers remains of strategic importance. Retaining this knowledge by formally capturing it is a challenge for industrial organisations. This paper explores research on automatically capturing this knowledge by using methods from activity recognition and process mining on data obtained from sensorised workers and environments. Activity recognition lifts the abstraction level of sensor data to recognizable activities and process mining methods discover models of process executions. We classify the existing work, which largely neglects the possibility of applying process mining, and derive a taxonomy that identifies challenges and research gaps.
\end{abstract}

Keywords: Activity recognition · Process mining · Manufacturing · Industrial environment $\cdot$ Tacit knowledge $\cdot$ Literature overview.

\section{Introduction}

The rise of the knowledge worker has contributed to the emphasis on the strategic value of creating, harnessing and applying knowledge within manufacturing environments. With the advent of automation, as part of the Industry 4.0 evolution, the strategic importance of knowledge and high skilled workers has only become more important. However, so did the crippling impact caused by knowledge gaps resulting from the difficulty of managing effectively tacit knowledge garnered through the experience of highly skilled workers once removed from their work environment. In fact, with the continuous advances in technology and increased complexity associated to both the product and the manufacturing processes, tacit knowledge represents by far the bulkiest part of an organization's knowledge. Many of the theories and methodologies associated with the externalization of tacit knowledge require organizational processes and a culture pervading the workplace that facilitate the creation of formal and external knowledge.

The digitization of the workplace through the pervasiveness of sensors, combined with ever more elaborate digital information systems, generates huge amounts of data that may be further enriched when considering the direct placement of sensors on workers in the shopfloor, thus capturing more effectively what is taking place as much of 


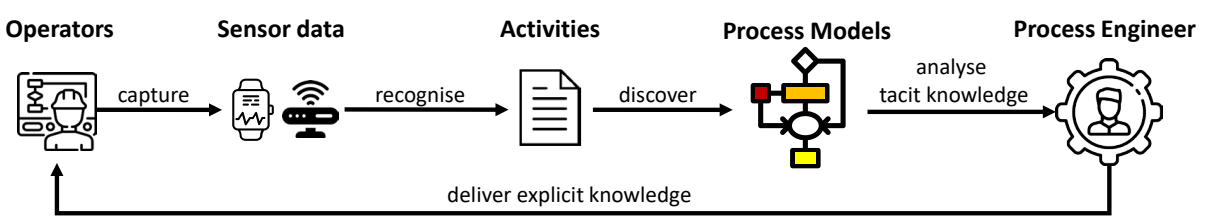

Fig. 1. Overview of the envisioned approach combining activity recognition and process mining.

the work entails manual activity, not registered in the supporting information system. With the wealth of data captured, including the human dimension, we envision the approach illustrated in Figure 1 as a way to externalise tacit knowledge of the operator on the shopfloor. The approach uses sensors and combines activity recognition [4] with process discovery, which automatically derives process models from activity execution sequences [1].

The purpose of this paper is to conduct a structured literature review on activity recognition applied in industrial contexts with the purpose of externalisation of tacit knowledge. In most, if not all cases, there is no automatic process discovery as the methods and approaches documented in literature are largely dependent on context with supervised learning. Those few unsupervised learning approaches rely on clustering techniques, largely ignoring the benefits of process mining in the discovery of process knowledge. The result of the synthesis of the literature review yielded a preliminary taxonomy to support the identification of challenges to be addressed, outlining potential areas of research to develop solutions that leverage activity recognition with process mining towards facilitating externalisation of tacit knowledge.

We structure the remainder as follows. In Section 2, activity recognition and process mining are briefly introduced. Section 3 presents our literature search. Based on the results, we present a preliminary taxonomy together with challenges in Section 4 We conclude the paper with an outlook for future work in Section 5

\section{Background}

We give a brief overview of activity recognition and process mining.

\subsection{Activity recognition}

Activity recognition (ARC) seeks to accurately identify human activities on various levels of granularity by using sensor readings. In recent years ARC has become an emerging field due to the availability of large amount of data generated by pervasive and ubiquitous computing [2|4|18]. Methods have demonstrated an increased efficiency in extracting and learning to recognise activities in the supervised learning setting using a range of machine learning techniques. Traditional methods often adopt shallow learning techniques such as Decision Trees, Naïve Bayes, Support Vector Machines (SVM), and Hidden Markov Models (HMM) [4] while the more recent methods often use Neural Network architectures, which require less manual feature engineering and exhibit better performance [29]39]. 
Applications of activity recognition span from smart home (behaviour analysis for assistance) to sports (automatic performances tracking and skill assessment) and even healthcare (medication tracking). The recognition of activities is not an end in itself, but often supports assistance, assessment, prediction and intervention related to the recognised activity. An emerging application field for ARC relates to smart factories and Industry 4.0 where an increasingly sensors-rich environment is generating large amounts of sensor data.

ARC captures activities through the use of sensors such as cameras, motion-sensors, and microphones. Despite the large amount of work, ARC remains a challenging problem due to the complexity and variability of activities as well as due to the context in which activities are meant to be recognised. Data labelling, for instance, is a common challenge related to ARC. Assigning the correct ground truth label is a very timeconsuming task. There has been less work on unsupervised [21] or semi-supervised techniques [19] which require fewer annotations [2]39]. Another challenge lies in the emergent topic of transfer learning [29], which helps with the redeployment of an ARC model from one factory floor to another with a different layout, environmental factors, population and activities.

\subsection{Process mining}

Process mining is a data analytics method that uses event logs to provide a data-driven view on the actual process execution for analysis and optimisation purposes [1]. Consider, e.g., the order-to-cash process of a manufacturing company. One execution of this process results in a sequence of events (or process trace) being recorded across several information systems. A process trace should contain at least the following: the activities names executed (e.g., order created) as well as their execution time. An event log is a set of process traces in which each process trace groups together the activities performed in one instance of a recurring process. Process mining can help to uncover the tacit process knowledge of workers by discovering process models from event logs. The discovered models reveal how work is actually performed, including deviations from standard procedures such as workarounds and re-work. Moreover, the actual process execution can be contrasted with existing de-jure models, e.g., to pinpoint deviations to work instructions and analyse performance issues. An in-depth introduction ot process discovery is given in [1] and [6] gives a comprehensive survey of process discovery methods.

However, only very few applications of process mining are reported within the manufacturing domain [13|23]. One reason for this gap might be that in many industrial environments, much of the manual work is not precisely captured in databases or logs. For example, the individual steps performed in an assembly task remain hidden when using event logs from standard information systems only. Thus, the recognition of such manually executed activities is a crucial prerequisite for the successful application of process mining in this context [16].

\section{Literature overview}

Based on the premise that activity recognition and process mining can be combined to extract tacit knowledge of operators in industrial processes, we conducted a search of 
Table 1. Results of the literature search classified according to the described criteria.

\begin{tabular}{|c|c|c|c|c|c|c|c|c|c|c|c|c|}
\hline & \multirow[t]{2}{*}{ Recognition } & \multirow[t]{2}{*}{ Time } & \multirow[t]{2}{*}{ Granularity } & \multirow[t]{2}{*}{ Context } & \multirow[t]{2}{*}{ Setting } & \multicolumn{4}{|c|}{ Sensor type } & \multicolumn{3}{|c|}{ S. location } \\
\hline & & & & & & $\mathrm{V}$ & $\mathrm{M}$ & $\mathrm{S}$ & $\mathrm{R}$ & $\mathrm{W}$ & $\mathrm{O}$ & A \\
\hline 20 & Supervised & Online & Fine & $x$ & Real & & $\checkmark$ & $\checkmark$ & & $\checkmark$ & & \\
\hline 99 & Supervised & Online & Fine & $x$ & $\mathrm{Lab}$ & & $\checkmark$ & & $\checkmark$ & $\checkmark$ & & $\checkmark$ \\
\hline 80 & Supervised & Online & Fine & $x$ & $\mathrm{Lab}$ & & $\checkmark$ & $\checkmark$ & & $\checkmark$ & & $\checkmark$ \\
\hline 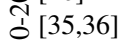 & Supervised & Online & Fine & State machine & Real & & $\checkmark$ & & $\checkmark$ & $\checkmark$ & $\checkmark$ & \\
\hline 24 & Supervised & Online & Fine & Sequence & Real & & & & $\checkmark$ & & & $\checkmark$ \\
\hline$\sqrt{28}$ & Supervised & Online & Coarse & $x$ & Real & & $\checkmark$ & & $\checkmark$ & $\checkmark$ & & $\checkmark$ \\
\hline 10 & Semi & Online & Both & Hierarchy & Lab & & $\checkmark$ & & & $\checkmark$ & & \\
\hline 25 & Semi & Post-mortem & Both & Sequence & Real & $\checkmark$ & & & & & & $\checkmark$ \\
\hline 27 & Supervised & Online & Fine & $x$ & Lab & & $\checkmark$ & & $\checkmark$ & $\checkmark$ & & $\checkmark$ \\
\hline 38 & Supervised & Online & Coarse & Workflow & Real & $\checkmark$ & & & & & & $\checkmark$ \\
\hline$=37$ & Supervised & Online & Both & $x$ & Real & $\checkmark$ & & & $\checkmark$ & & & $\checkmark$ \\
\hline ণิ [14] & Supervised & Predictive & Fine & State machine & Lab & $\checkmark$ & & & & & & $\checkmark$ \\
\hline$\stackrel{2}{2}$ & Supervised & Online & Coarse & Workflow & $x$ & & & & $\checkmark$ & & $\checkmark$ & \\
\hline ㄱ 32 32 33 & Supervised & Online & Both & Hierarchy & Lab & $\checkmark$ & $\checkmark$ & & & & & $\checkmark$ \\
\hline 8 & Supervised & Post-mortem & Fine & Probabilistic & $x$ & & & & & & & \\
\hline 34 & Supervised & Predictive & Fine & Rules & Lab & $\checkmark$ & & & & & & $\checkmark$ \\
\hline 26 & Supervised & Online & Fine & Sequence & Lab & $\checkmark$ & $\checkmark$ & & & & & $\checkmark$ \\
\hline ○ 21 & Unsupervised & Online & Coarse & Workflow & Real & $\checkmark$ & $\checkmark$ & & & $\checkmark$ & & $\checkmark$ \\
\hline [12 & Supervised & Online & Fine & $x$ & Real & & $\checkmark$ & & & $\checkmark$ & & \\
\hline مَ & Supervised & Online & Coarse & $x$ & Real & & $\checkmark$ & & & $\checkmark$ & & \\
\hline 8 & Unsupervised & Post-mortem & Coarse & Sequence & Lab & & $\checkmark$ & & & $\checkmark$ & & \\
\hline \17 & Supervised & Online & Fine & $x$ & Lab & $\checkmark$ & & & & & & 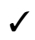 \\
\hline 30 & Supervised & Online & Fine & Workflow & Real & & $\checkmark$ & & & $\checkmark$ & & \\
\hline 5 & Semi & Online & Both & Rules & $\mathrm{Lab}$ & $\checkmark$ & $\checkmark$ & & & $\checkmark$ & & \\
\hline
\end{tabular}

the existing literature on activity recognition in industrial environments. Our goal was to derive a taxonomy that helps to identify the central issues and challenges of using activity recognition and process discovery for externalizing tacit knowledge.

We searched both Google Scholar and Scopus for research on activity recognition that was applied in or is applicable to industrial settings. We used the keywords events or sensors, activity recognition, industrial or manufacturing in our search and followedup references in the identified work. Furthermore, we widened our search by looking for research on activity recognition that mentions one of the keywords tacit knowledge, process discovery, process elicitation, process analysis. An initial search revealed that ARC can be decomposed into conceptual work and applied work. For example, in [31] a architecture for process mining in cyber-physical systems is proposed but was not evaluated. Although such work provides useful insights, they have not be validated and might not be applicable in real-work environments. Therefore, we excluded purely conceptual work. Furthermore, we excluded work without connection to an industrial setting. We identified 26 relevant papers that are listed in Table 1 . We do not believe our literature review is exhaustive, but we do believe it is representative of existing literature.

We classified the work according to the following criteria. 
- Its recognition type based on the kind of prior knowledge employed into methods for supervised recognition, unsupervised recognition, and semi-supervised recognition.

- The time horizon of the recognition was categorised into predictive, online, and post-mortem recognition.

- We distinguished the sensor type into vision-based (V), motion-based (M), soundbased (S), and radiowave-based (R) sensors. Note that if a RGB camera (visionbased) is used to determine worker movement, we consider it both as vision-based and motion-based sensor.

- Regarding the sensor location, we categorise sensors into those attached to objects $(\mathrm{O})$, those ambient in the environment (A), and those wearable (W).

- There is a large variety of activities in industrial settings some of which are more difficult to detect. We categorized the work based on the supported granularity of the activities into: coarse and fine. An example of activity recognition on a coarse granularity level would be recognising that a part of the assembly was installed, whereas on a fine level of granularity recognition would recognise the individual steps required to connect that part, e.g., pick-up screw and fasten screw. Some approaches support both coarse-grained and fine-grained activities.

- We also distinguished whether the work takes the context of the assembly process into account to improve the detection, e.g., by making use of existing assembly instructions in form of higher-level workflow models, state machines, sequences, or other models.

- Lastly, we distinguished the setting in which the method was evaluated into artificial laboratory settings or in real factory environments.

\section{Taxonomy for activity recognition and process discovery in industrial environments}

Based on our literature study, we derived a taxonomy for knowledge extraction through activity recognition in industrial environments. The taxonomy focuses on the applicability in practical settings and the requirements on activity recognition in a processmining context. Our goal is to identify challenges for the joint application and help designing new systems for knowledge extraction by describing existing systems in a unified manner. The taxonomy is organised around four major dimensions: time, data, process context, environment, and privacy. We acknowledge that the taxonomy is still under development. Therefore, we only briefly sketch each of the dimensions with examples from the literature.

Time. In Table 1, we distinguished three major categories of activity recognition regarding the time dimension: predictive, online, and post-mortem activity recognition. Most activity recognition methods in the industrial setting target the online setting, in which the activity is detect during its execution. This can be useful to provide up-todate information for the activity at hand, e.g., in [35] a check list is kept updated. We found much fewer examples for the predictive setting, in which the next activity is predicted before or just when it is about to happen also denoted as intention recognition. 
A notable exception is [34] which uses state recognition to predict the next activity in a manufacturing application. Such predictive recognition can be useful to provide timely assistance to prevent errors. Lastly, post-mortem activity recognition methods can use both information about past activities as well as future activities to determine the most likely classification. Only two methods in Table 1 take the post-mortem view on activity recognition. This shows that the tacit knowledge discovery angle has been largely neglected. The work in [8] is an exception and, indeed, conceptually close to work on conformance checking and the optimal alignment of event sequences to process models in the process mining literature [22]. Thus, there are clear research gaps regarding the predictive and post-mortem category of activity recognition in industrial contexts (such as manufacturing) out of which the post-mortem angle is more relevant for our envisioned approach.

Data. The availability of data is a crucial prerequisite for externalising tacit knowledge through process mining and activity recognition. There are several categories in the data dimension: capture, storage and processing of data. Several challenges have to be dealt with in our application scenario. We exemplify one challenge regarding the data processing category. Here the availability of ground truth labels is a particular challenge. Since the goal of process discovery is, in fact, to discover the unknown tacit knowledge of workers, it is questionable whether all the activity labels for the use of supervised methods can be determined beforehand. However, as clear from Table 1 there have been only very few unsupervised techniques proposed.

Process context. Several factors are relevant to the process context dimension, such as the type of activities executed, the type of control-flow in which the activities are embedded, and their complexity. For example, Bader et al [8] mentions the challenge of considering the teamwork setting in which some activities are of a collaborative nature: multiple workers collaborate on one activity. However, they do not yet provide a solution. Also relevant to the process-context dimension is that some work takes into account a-priori knowledge on the control-flow of the process. For example, in [36] a finite state machine is used to encode this prior knowledge whereas in [7] a higher-level process modelling language is used to define the process. An opportunity for future work might be to leverage on the wealth of higher-level modelling notations used in a process mining context [1]. Lastly the complexity of the considered processes and of the activities is worth discussion. In most settings only a few activities are considered (less than 10) and only few consider hierachical dependencies between activities on lower and higher levels. More advanced work in this category are the semi-supervised techniques in [5|10] in which higher-level activities are recognized based on sequences of detected low-level activities.

Environment. The environment in which the activities take place is highly relevant to the practical applicability of extracting tacit knowledge through activity recognition and process discovery. For example, the sensor type needs to be carefully selected since there are often several restrictions in a real factory setting [3]: wearable sensors should not interfere with the actual work and safety protocols and ambient sensors are 
often limited to narrow areas or subject to background noise. Some of the work identified evaluated their method in a realistic factory environment. However, the evaluation mostly takes place in designated areas to avoid costly interruption of production lines. For example, in [36] car assembly activities in a Skoda factory are tracked, but only in a "learning island" that is used for training workers. Thus, the applicability of many techniques on a real production line remains unclear.

Privacy. Activity recognition requires the capture of data, which may include sensors on the operator themselves. This raises important concerns with regards to privacy as the use of the data may have a negative impact on the operator (e.g.: due to poor performance, an operators employment is terminated). The body of research covered, with exception of [3], focuses very much on the opportunities of processing the data collated, whilst disregarding the potential threats to the operators well-being [23]. To address the challenges, governments have intervened wtih regulatory frameworks to safeguard the privacy of the user, such as the General Data Protection Regulation (GDPR) that attempts to place the user in control of their digital selves. Therefore, privacy has become a design requirement and not an afterthought, which may affect how activity recognition research may be realised.

\section{Conclusion}

We presented a structured literature review on activity recognition from the viewpoint of using the recognised activity data as input to process discovery techniques to reveal tacit knowledge of industrial operators. Based on the identified literature, we contribute a preliminary taxonomy for knowledge extraction from manual industrial processes through activity recognition. Whereas we believe to have included the most relevant literature from the field of activity recognition, we acknowledge that, as future work, this study should be further extended to take into account research from the field of learning organisations and look in more depth at the process discovery task after having recognised relevant activities.

Acknowledgments. This research has received funding from the European Unions H2020 research and innovation programme under grant agreement no. 723737 (HUMAN).

\section{References}

1. van der Aalst, W.M.P.: Process Mining - Data Science in Action, Second Edition. Springer (2016)

2. Abdallah, Z.S., Gaber, M.M., Srinivasan, B., Krishnaswamy, S.: Activity recognition with evolving data streams. ACM Computing Surveys 51(4), 1-36 (2018)

3. Aehnelt, M., Gutzeit, E., Urban, B.: Using activity recognition for the tracking of assembly processes: Challenges and requirements. In: WOAR 2014. Fraunhofer Verlag (2014)

4. Aggarwal, J., Ryoo, M.: Human activity analysis. ACM Computing Surveys 43(3), 1-43 (2011) 
5. Al-Naser, M., Ohashi, H., Ahmed, S., Nakamura, K., Akiyama, T., Sato, T., Nguyen, P.X., Dengel, A.: Hierarchical model for zero-shot activity recognition using wearable sensors. In: ICAART (2). pp. 478-485. SciTePress (2018)

6. Augusto, A., Conforti, R., Dumas, M., Rosa, M.L., Maggi, F.M., Marrella, A., Mecella, M., Soo, A.: Automated discovery of process models from event logs: Review and benchmark. IEEE Transactions on Knowledge and Data Engineering (2018)

7. Bader, S., Aehnelt, M.: Tracking assembly processes and providing assistance in smart factories. In: ICAART 2014. SCITEPRESS (2014)

8. Bader, S., Krger, F., Kirste, T.: Computational causal behaviour models for assisted manufacturing. In: iWOAR 2015. ACM Press (2015)

9. Bannach, D., Kunze, K.S., Lukowicz, P., Amft, O.: Distributed modular toolbox for multimodal context recognition. In: ARCS. LNCS, vol. 3894, pp. 99-113. Springer (2006)

10. Blanke, U., Schiele, B.: Remember and transfer what you have learned - recognizing composite activities based on activity spotting. In: ISWC 2010. IEEE (2010)

11. Böttcher, S., Scholl, P.M., Laerhoven, K.V.: Detecting process transitions from wearable sensors. In: iWOAR 2017. ACM Press (2017)

12. Feldhorst, S., Masoudenijad, M., ten Hompel, M., Fink, G.A.: Motion classification for analyzing the order picking process using mobile sensors - general concepts, case studies and empirical evaluation. In: ICPRAM 2016. pp. 706-713. SCITEPRESS (2016)

13. Gonella, P., Castellano, M., Riccardi, P., Carbone, R.: Process mining: A database of applications. Tech. rep., HSPI SpA - Management Consulting (2017)

14. Goto, H., Miura, J., Sugiyama, J.: Human-robot collaborative assembly by on-line human action recognition based on an FSM task model. In: Human-Robot Interaction 2013 Workshop on Collaborative Manipulation (2013)

15. Grzeszick, R., Lenk, J.M., Rueda, F.M., Fink, G.A., Feldhorst, S., ten Hompel, M.: Deep neural network based human activity recognition for the order picking process. In: iWOAR 2017. ACM Press (2017)

16. Janiesch, C., Koschmider, A., Mecella, M., Weber, B., Burattin, A., Ciccio, C.D., Gal, A., Kannengiesser, U., Mannhardt, F., Mendling, J., Oberweis, A., Reichert, M., RinderleMa, S., Song, W., Su, J., Torres, V., Weidlich, M., Weske, M., Zhang, L.: The internetof-things meets business process management: Mutual benefits and challenges (2017), arXiv: 1709.03628

17. Knoch, S., Ponpathirkoottam, S., Fettke, P., Loos, P.: Technology-enhanced process elicitation of worker activities in manufacturing. In: Business Process Management Workshops, pp. 273-284. Springer International Publishing (2018)

18. Lara, O.D., Labrador, M.A.: A survey on human activity recognition using wearable sensors. IEEE Communications Surveys \& Tutorials 15(3), 1192-1209 (2013)

19. Longstaff, B., Reddy, S., Estrin, D.: Improving activity classification for health applications on mobile devices using active and semi-supervised learning. In: ICST 2010. IEEE (2010)

20. Lukowicz, P., Ward, J.A., Junker, H., Stäger, M., Tröster, G., Atrash, A., Starner, T.: Recognizing workshop activity using body worn microphones and accelerometers. In: Ferscha, A., Mattern, F. (eds.) PERVASIVE 2004. LNCS, vol. 3001, pp. 18-32. Springer (2004)

21. Maekawa, T., Nakai, D., Ohara, K., Namioka, Y.: Toward practical factory activity recognition. In: UbiComp 2016. ACM Press (2016)

22. Mannhardt, F., de Leoni, M., Reijers, H.A., van der Aalst, W.M.P.: Balanced multiperspective checking of process conformance. Computing 98(4), 407-437 (2016)

23. Mannhardt, F., Petersen, S.A., de Oliveira, M.F.D.: Privacy challenges for process mining in human-centered industrial environments. In: Intelligent Environments (IE) 2018. IEEE Xplore (To Appear) 
24. Marin-Perianu, M., Lombriser, C., Amft, O., Havinga, P., Trster, G.: Distributed activity recognition with fuzzy-enabled wireless sensor networks. In: DCOSS 2008, pp. 296-313. Springer (2008)

25. Mrzinger, R., Thallinger, G., Anagnostopoulos, V., Kosmopoulos, D., Voulodimos, A., Lalos, C., Doulamis, N., Varvarigou, T., Zelada, R.P., Soler, I.J., Stalder, S., Sardis, M., Gool, L.V., Middleton, L., Sabeur, Z., Arbab-Zavar, B., Carter, J., Nixon, M., Rosenberg, I., Grabner, H., Veres, G., Bouchrika, I., Thaler, M., Schuster, R., Hofmann, A.: Tools for semi-automatic monitoring of industrial workflows. In: ARTEMIS 2010. ACM Press (2010)

26. Mura, M.D., Dini, G., Failli, F.: An integrated environment based on augmented reality and sensing device for manual assembly workstations. Procedia CIRP 41, 340-345 (2016)

27. Ogris, G., Lukowicz, P., Stiefmeier, T., Trster, G.: Continuous activity recognition in a maintenance scenario: combining motion sensors and ultrasonic hands tracking. Pattern Analysis and Applications 15(1), 87-111 (2011)

28. Ogris, G., Stiefmeier, T., Lukowicz, P., Troster, G.: Using a complex multi-modal on-body sensor system for activity spotting. In: IWSC 2008. IEEE (2008)

29. Ramamurthy, S.R., Roy, N.: Recent trends in machine learning for human activity recognition-a survey. Wiley Interdiscip Rev Data Min Knowl Discov 8(4), e1254 (2018)

30. Raso, R., Emrich, A., Burghardt, T., Schlenker, M., Gudehus, T., Strter, O., Fettke, P., Loos, P.: Activity monitoring using wearable sensors in manual production processes - an application of cps for automated ergonomic assessments. In: MKWI 2018. Leuphana Universitt Lneburg (2018)

31. Repta, D., Moisescu, M.A., Sacala, I.S., Stanescu, A.M., Constantin, N.: Generic architecture for process mining in the context of cyber physical systems. Applied Mechanics and Materials 656, 569-577 (2014)

32. Roitberg, A., Perzylo, A., Somani, N., Giuliani, M., Rickert, M., Knoll, A.: Human activity recognition in the context of industrial human-robot interaction. In: APSIPA 2014. IEEE (2014)

33. Roitberg, A., Somani, N., Perzylo, A., Rickert, M., Knoll, A.: Multimodal human activity recognition for industrial manufacturing processes in robotic workcells. In: ICMI 2015. ACM Press (2015)

34. Schlenoff, C., Kootbally, Z., Pietromartire, A., Franaszek, M., Foufou, S.: Intention recognition in manufacturing applications. Robot Comput Integr Manuf 33, 29-41 (2015)

35. Stiefmeier, T., Lombriser, C., Roggen, D., Junker, H., Ogris, G., Troester, G.: Event-based activity tracking in work environments. In: IFAWC 2006. pp. 1-10 (2006)

36. Stiefmeier, T., Roggen, D., Ogris, G., Lukowicz, P., Tr, G.: Wearable activity tracking in car manufacturing. IEEE Pervasive Computing 7(2), 42-50 (2008)

37. Voulodimos, A., Kosmopoulos, D., Vasileiou, G., Sardis, E., Anagnostopoulos, V., Lalos, C., Doulamis, A., Varvarigou, T.: A threefold dataset for activity and workflow recognition in complex industrial environments. IEEE Multimedia 19(3), 42-52 (2012)

38. Voulodimos, A.S., Kosmopoulos, D.I., Doulamis, N.D., Varvarigou, T.A.: A top-down eventdriven approach for concurrent activity recognition. Multimedia Tools and Applications 69(2), 293-311 (2012)

39. Wang, J., Chen, Y., Hao, S., Peng, X., Hu, L.: Deep learning for sensor-based activity recognition: A survey. Pattern Recognition Letters (2018)

40. Ward, J.A., Lukowicz, P., Troster, G., Starner, T.E.: Activity recognition of assembly tasks using body-worn microphones and accelerometers. IEEE Trans Pattern Anal Mach Intell 28(10), 1553-1567 (2006) 